\title{
CONCORDANCES OF HILBERT CUBE MANIFOLDS
}

\author{
BY \\ T. A. CHAPMAN( $\left.{ }^{1}\right)$
}

\begin{abstract}
The main result of this paper asserts that homotopy groups of concordances of compact Hilbert cube manifolds are isomorphic to homotopy groups of concordances of compact finite-dimensional piecewise-linear manifolds. This enables us to apply some finite-dimensional results to obtain some new information about homotopy groups of homeomorphism groups of compact Hilbert cube manifolds. Our approach also yields a much shorter proof of the local contractibility of the homeomorphism group of any compact Hilbert cube manifold.
\end{abstract}

1. Introduction. If $X$ is a compact metric space, then we will use $C(X)$ to denote the space of all concordances of $X$, i.e. $C(X)$ is the function space of all homeomorphisms of $[0,1] \times X$ onto itself which fix $0 \times X$. We give $C(X)$ the compact-open topology and let it be pointed, with base point the identity. $Q$ is used to denote the Hilbert cube, which is the countable-infinite product of closed intervals $[-1,1]$, and a $Q$-manifold is a separable metric manifold modeled on $Q$.

We first recall some facts from [7]. The following is the main result established there.

(A) If $M$ is a compact PL manifold, then there exists an $i \geqslant 0$ and a surjection $(\times \text { id })_{*}: \pi_{0} C\left(M \times[-1,1]^{i}\right) \rightarrow \pi_{0} C(M \times Q)$, where $\times$ id: $C\left(M \times[-1,1]^{i}\right)$ $\rightarrow C(M \times Q)$ is obtained by crossing each homeomorphism with the identity.

(Recall that $\pi_{0} C(X)$ is the set of path components of $C(X)$. It becomes a group under composition.) This result just says that any homeomorphism $h \in$ $C(M \times Q)$ is isotopic, rel $0 \times M \times Q$, to a homeomorphism of the form $f \times$ id, where $f \in C\left(M \times[-1,1]^{t}\right)$ and $[-1,1]^{i}$ is regarded as the product of the first $i$ intervals in $Q=[-1,1]^{\infty}$. The proof of (A) uses standard tools from PL manifold theory along with the following stability result of [10]: If $M$ is a compact $P L$ manifold, then the homomorphism ( $\times$ id $)_{*}: \pi_{n} C(M) \rightarrow \pi_{n} C(M \times[-1,1])$ is an isomorphism, for $\operatorname{dim} M \gg n$. Without this stability result, (A) asserts that there is a surjection of the direct limit of

Received by the editors November 22, 1974.

AMS (MOS) subject classifications (1970). Primary 57A20; Secondary 55E99.

Key words and phrases. $Q$-manifold, concordance, homeomorphism group, group of self-equivalences.

$\left({ }^{1}\right)$ The author is an A. P. Sloan Fellow and is supported in part by NSF Grant GP-28374.

Copyright $\odot$ 1976, American Mathematical Society 


$$
\pi_{0} C(M) \stackrel{(\times \mathrm{id})_{*}}{\longrightarrow} \pi_{0} \mathrm{C}(M \times[-1,1]) \stackrel{(\times \mathrm{id})_{*}}{\longrightarrow} \pi_{0} \mathrm{C}\left(M \times[-1,1]^{2}\right) \rightarrow \cdots
$$

onto $\pi_{0} C(M \times Q)$.

Using (A) above we have a means of obtaining information about homeomorphisms on $M \times Q$ from homeomorphisms on $M \times[-1,1]^{i}$. In particular, the following result is a corollary of (A).

(B) If $M$ is a compact 1-connected PL manifold, then $\pi_{0} C(M \times Q)=0$.

For this we use the fact that $\pi_{0} C(M)=0$, for $M 1$-connected and $\operatorname{dim} M \geqslant$ 5 [13]. Using a little $Q$-manifold theory this implies (again from [7]) that any two homotopic homeomorphisms on a compact 1-connected $Q$-manifold are ambient isotopic. This result is not surprising if one recalls the parallel situation for $l_{2}$-manifolds, where $l_{2}$ is separable Hilbert space. In [1] it was shown that any two homotopic homeomorphisms on an $l_{2}$-manifold are ambient isotopic (see [4] for a shorter proof). The surprising part of (B) above is the difficulty of its proof.

It was also shown in [7] that the assumption of 1-connectivity in (B) above cannot always be dropped. Here is the result.

(C) For some $i>0$ we have an isomorphism $(\times \mathrm{id})_{*}: \pi_{0} \mathrm{C}\left(S^{1} \times I^{\prime}\right) \approx$ $\pi_{0} C\left(S^{1} \times Q\right)$. It follows from the PL Pseudo-Isotopy Theorem of [11] that $\pi_{0} C\left(S^{1} \times I^{i}\right) \neq 0$, for $\left.i \geqslant 5 .{ }^{2}\right)$ Using this result, along with (C) and a little $Q$-manifold theory (again see [7]), we conclude that there exist homeomorphisms on $S^{1} \times Q$ which are homotopic but not ambient isotopic.

The purpose of this paper is to prove the following result.

THEOREM 1. If $M$ is a compact PL manifold, then for each $n \geqslant 0$ there exists an $i \geqslant 0$ (depending on $n$ ) and an isomorphism $(\times \text { id })_{*}: \pi_{n} C\left(M \times[-1,1]^{t}\right)$ $\approx \pi_{n} \mathrm{C}(M \times Q)$.

This amounts to a substantial improvement of (A) and (C) above. In fact this completely reduces the problem of the calculation of the homotopy groups of $C(X)$, for $X$ a compact $Q$-manifold, to the corresponding "stable" problem for compact PL manifolds (because $X$ is homeomorphic to some $M \times Q$ [5]). Here is an immediate consequence of Theorem 1.

THEOREM 2. If $X$ is a compact connected Q-manifold, then $\pi_{0} C(X)=0$ iff $X$ is 1-connected.

Theorem 2 gives us the other half of (B) above. It is deduced from Theorem 1 by using the following consequence of the PL Pseudo-Isotopy Theorem of [11]: $\pi_{0} C(M)=0$ iff $M$ is 1-connected, for $M$ a compact connected $P L$ manifold, $\operatorname{dim} M \geqslant 5$.
$\left({ }^{2}\right)$ In fact $\pi_{0} C\left(S^{1} \times I^{i}\right) \approx z_{2} \oplus Z_{2} \oplus \cdots \cdot$ 
Concerning the higher homotopy groups we have the following less precise result.

THEOREM 3. Let $X, Y$ be compact, connected $Q$-manifolds and let $f$ : $X \rightarrow Y$ be a $k$-connected map, $k \geqslant 2$. Then there is induced an isomorphism $\pi_{n} C(X) \approx \pi_{n} C(Y)$, for $0 \leqslant n \leqslant k-2$.

Applying this to the case $Y=Q$ we have the following

COROLlarY. If $X$ is a compact $Q$-manifold which is $k$-connected, $k \geqslant 2$, then $\pi_{n} C(X)=0$, for $0 \leqslant n \leqslant k-2$.

Theorem 3 is deduced from Thoerem 1 by use of the following PL result of [10]: If $f: M \rightarrow N$ is a $k$-connected map of compact, connected PL manifolds, where $\operatorname{dim} M, \operatorname{dim} N \gg k \geqslant 2$, then there is induced an isomorphism $\pi_{n} C(M) \approx \pi_{n} C(N)$, for $0 \leqslant n \leqslant k-2$.

The above results also shed some light on the homotopy groups of homeomorphism groups of $Q$-manifolds. For any compact metric space $X$ let $H(X)$ denote its homeomorphism group and let $E(X)$ denote its space of all self-homotopy equivalences. We give each space the compact-open topology and let it be pointed, with base point the identity. Here is our result concerning homeomorphism groups. For more details see $\$ 7$.

THEOREM 4. If $X$ is a compact $Q$-manifold which is $k$-connected, $k \geqslant 2$, then the inclusion $H(X) \hookrightarrow E(X)$ induces an isomorphism $\pi_{n} H(X) \approx \pi_{n} E(X)$, for $0 \leqslant n \leqslant k-2$, and an epimorphism for $n=k-1$.

We now make a few comments concerning the organization of the material in this paper. In $\$ 2$ we introduce some notation and then we prove a result (Theorem 2.1) which will be needed in $\$ 4$ for the proof of Theorem 4.1. Incidentally, Theorem 2.1 gives us a somewhat easier proof of the local contractibility of the homeomorphism group of a compact $Q$-manifold [3]: this is carried out in Theorem 2.2. The main technical result needed in the proof of Theorem 1 is the Representation Theorem. It is stated in $\$ 3$ and is proved in $\$ \S 4-5$. Then in $\$ 6$ we prove Theorem 1 and in $\$ 7$ we prove Theorem 4.

2. A key result. In this section we introduce some notation and then prove a result (Theorem 2.1 ) which will be used in the proof of Theorem 4.1. We also prove (Theorem 2.2 ) that the homeomorphism group of any compact $Q$-manifold is locally contractible. This is a significant simplification of the argument given in [3].

We use $R^{n}$ to denote euclidean $n$-space and for any $r>0$ we let $B_{r}^{n}=$ $[-r, r]^{n} \subset R^{n}$. The interior and boundary of $B_{r}^{n}$ will be denoted by $\stackrel{\circ}{r}_{r}^{n}$ and 
$\partial B_{r}^{n}$, respectively, while the prefixes Int and Bd will be used to denote topological interior and boundary in general. If $r=1$ we will omit the subscript on $B_{r}^{n}$. We also let $I=[0,1]$ and $\Delta$ will be used to denote a standard simplex in $R^{n}$.

For spaces $X, Y$, and $Z$ a homeomorphism $h: X \times Y \rightarrow X \times Z$ is said to be $X$-preserving provided that $h(x \times Y)=x \times Z$, for each $x \in X$. For each $x \in X$ we use $h_{x}: Y \rightarrow Z$ for the level of $h$ defined by $h_{x}(y)=z$, where $h(x, y)$ $=(x, z)$. If $g: X \times Y \rightarrow X \times Z$ is another $X$-preserving homeomorphism, then we write $h \stackrel{\text { iso }}{\simeq}(X) g$ to mean that $h$ is isotopic to $g$, with each level of the isotopy being $X$-preserving. Of course the notation $h \stackrel{\text { iso }}{\simeq}(X) g$ rel $A$ has the usual meaning. The symbol "id" will be used to denote the identity map of a space onto itself and id will also be (incorrectly) used to denote the inclusion map of a space into a larger space.

We are now ready for Theorem 2.1 .

THEOREM 2.1. Let $h: I \times \stackrel{\circ}{B}_{3}^{n} \times Q \rightarrow I \times R^{n} \times Q$ be an open embedding such that $h=\mathrm{id}$ on $0 \times \stackrel{\circ}{B}_{3}^{n} \times Q$. If $h$ is sufficiently close to id, then there exists a homeomorphism $\tilde{h}: I \times R^{n} \times Q \rightarrow I \times R^{n} \times Q$ such that $\tilde{h}=h$ on $I \times$ $B^{n} \times Q, \tilde{h}=$ id on $0 \times R^{n} \times Q$, and $\tilde{h}$ is supported on $h\left(I \times B_{2}^{n} \times Q\right)$. Moreover $\tilde{h}$ depends continuously on $h$ and $\tilde{h}=$ id if $h=\mathrm{id}$.

Proof. To save time we will assume that the reader is familar with the technique described in $[9, \S 8]$ for wrapping small homeomorphisms around tori. Then applying the main diagram (as used in [3]) we can choose $h$ sufficiently close to id so that we can find a homeomorphism $\hat{h}: I \times R^{n} \times Q \rightarrow I \times R^{n} \times Q$ such that

(1) $\hat{h}=h$ on $I \times B^{n} \times Q$,

(2) $\hat{h}=$ id on $0 \times R^{n} \times Q$,

(3) $\hat{h}$ is bounded, i.e. $\left\{\left\|x_{1}-x_{2}\right\| \operatorname{lh}\left(t_{1}, x_{1}, q_{1}\right)=\left(t_{2}, x_{2}, q_{2}\right)\right\}$ is bounded above,

(4) $\hat{h}$ depends continuously on $h$,

(5) $\hat{h}=$ id if $h=$ id.

The construction of $\hat{h}$ uses no infinite-dimensional topology and can be carried out by anyone familar with [3] or [9].

Now choose $h$ sufficiently close to id so that

$$
h\left(I \times B^{n} \times Q\right) \subset I \times \stackrel{\circ}{B}_{1.5}^{n} \times Q \subset I \times B_{1.6}^{n} \times Q \subset h\left(I \times \stackrel{\circ}{B}_{2}^{n} \times Q\right) .
$$

Let $\alpha: \stackrel{\circ}{B}_{1.6}^{n} \rightarrow R^{n}$ be a radially-defined homeomorphism such that $\alpha=$ id on $B_{1.5}^{n}$ : Then $h_{1}=(\mathrm{id} \times \alpha \times \mathrm{id})^{-1} \hat{h}(\mathrm{id} \times \alpha \times \mathrm{id})$ defines a homeomorphism of $I \times \stackrel{\circ}{B}_{1.6}^{n} \times Q$ onto itself such that

(1) $h_{1}=h$ on $I \times B^{n} \times Q$, 
(2) $h_{1}=$ id on $0 \times B_{1.6}^{n} \times Q$,

(3) for each $\epsilon>0$ there exists an $r<1.6$ such that $\|p h(t, x, q)-x\|<\epsilon$, for all $(t, x, q) \in I \times\left(\stackrel{\circ}{B}_{1.6}^{n}-B_{r}^{n}\right) \times Q$, where $p: I \times \stackrel{\circ}{B}_{1.6}^{n} \times Q \rightarrow \dot{B}_{1.6}^{n}$ is the projection map,

(4) $h_{1}$ depends continuously on $h$,

(5) $h_{1}=$ id if $h=$ id.

Let the cone over $Q$ be represented by $C(Q)=(Q \times[0,1)) \cup\{v\}$ with the usual topology. Then there exists a homeomorphism $\beta: Q \times[0,1] \rightarrow C(Q)$ such that $\beta(Q \times 0)=Q \times 0$. This just follows from the fact that $Q$ is homeomorphic to its own cone and the Homogeneity Theorem (see [8]). Define

$$
\begin{gathered}
A=\left\{(x, q, t) \in R^{n} \times C(Q) \mid 1.5 \leqslant\|x\|<1.6 \text { and } 10(\|x\|-1.5) \leqslant t<1\right\} \\
\cup\left(\stackrel{\circ}{1.6}_{1.6}^{n}-\stackrel{\circ}{B}_{1.5}^{n}\right) \times\{v\} \subset\left(\stackrel{\circ}{1.6}_{1.6}^{n}-\stackrel{\circ}{B}_{1.5}^{n}\right) \times C(Q)
\end{gathered}
$$

and let $\gamma: A \rightarrow\left(\stackrel{\circ}{B}_{1.6}^{n}-\stackrel{\circ}{B}_{1.5}^{n}\right) \times C(Q)$ be defined by $\gamma(x, v)=(x, v)$ and by linearly homeomorphing $x \times q \times[10(\|x\|-1.5)$, 1) onto $x \times q \times[0,1)$, for each $x$ and $q$. Define $\theta: I \times\left(B_{1.6}^{n}-B_{1.5}^{n}\right) \times Q \rightarrow\left(B_{1.6}^{n}-B_{1.5}^{n}\right) \times C(Q)$ by $\theta(t, x, q)$ $=(x, \beta(q, t))$ and define $\delta:\left(I \times B_{1.5}^{n} \times Q\right) \cup \theta^{-1}(A) \rightarrow I \times B_{1.6}^{n} \times Q$ by setting $\delta=$ id on $I \times B_{1.5}^{n} \times Q$ and $\delta=\theta^{-1} \gamma \theta$ on $\theta^{-1}(A)$. Then $\delta^{-1} h_{1} \delta$ extends via the identity to $\tilde{h}$, a homeomorphism of $I \times R^{n} \times Q$ onto itself which fulfills our requirements.

We now use Theorem 2.1 to prove the main result of [3]. Note that we do not use the triangulation and classification theorems for $Q$-manifolds.

THEOREM 2.2. If $X$ is a compact $Q$-manifold, then the homeomorphism group of $X$ is locally contractible.

Proof. Using the fact that $X$ is homeomorphic to $I \times X$, all we have to do is prove that the homeomorphism group $H(I \times X)$ is locally contractible. Note that $C(X)$, the group of concordances of $X$, is a subspace of $H(I \times X)$. The first step will be to prove that $C(X)$ is locally contractible; then the next step is to use this fact to prove that $H(I \times X)$ is locally contractible.

I. $C(X)$ is locally contractible. Just as in [5] we can find a finite sequence $X=X_{0} \subset X_{1} \subset \cdots \subset X_{k-1} \subset X_{k}$, where each $X_{i}$ is a compact $Q$-manifold, $X_{k}$ is homeomorphic to $Q$, and for each $i \geqslant 1$ there exists an open embedding $h_{i}$ : $R^{n_{i}} \times Q \rightarrow X_{i}$ such that $X_{i-1}=X_{i}-h_{i}\left(B^{n_{i}} \times Q\right)$. Using the fact that $(I \times Q$, $0 \times Q$ ) is homeomorphic to (cone on $Q$, base) by the homeomorphism $\beta$ used in the proof of Theorem 2.1, and then applying the Alexander trick, it is easy to see that $C(Q)$ is locally contractible; therefore $C\left(X_{k}\right)$ is locally contractible. Working inductively backwards through the above sequence we will prove that the local contractibility of $C\left(X_{i}\right)$ implies the local contractibility of $C\left(X_{i-1}\right)$, for each $i \geqslant 1$. 
This will imply that $C(X)$ is locally contractible. So choose $i \geqslant 1$ and $h \in$ $\mathrm{C}\left(X_{i-1}\right)$. We will prove that if $h$ is sufficiently close to id, then $h \stackrel{\text { iso }}{\approx}$ id rel $0 \times$ $X_{i-1}$. It will follow from the construction that this isotopy depends continuously on $h$ and if $h=$ id, then each level of this isotopy is the identity. If we can do this, then it will clearly follow that $C\left(X_{i-1}\right)$ is locally contractible.

It is elementary to construct an isotopy $\phi_{t}: I \times X_{i-1} \rightarrow I \times X_{i-1}, 0 \leqslant$ $t \leqslant 1$, such that $\phi_{0}=\mathrm{id}, \phi_{t}\left(0 \times X_{i-1}\right) \subset 0 \times X_{i-1}$, for each $t$, and

$$
\phi_{1}\left(\left(0 \times X_{i-1}\right) \cup\left(I \times h_{i}\left(\partial B^{n_{i}} \times Q\right)\right)\right)=0 \times X_{i-1} .
$$

Then put $g_{t}=\phi_{t}^{-1} h \phi_{t}$, which defines an isotopy of $I \times X_{i-1}$ onto itself such that $h \stackrel{\text { iso }}{\simeq} g_{1}$ rel $0 \times X_{i-1}$ and $g_{1}=$ id on $I \times h_{i}\left(\partial B^{n_{i}} \times Q\right)$. By using a simple variation of the Alexander trick we may additionally assume that $g_{1}=$ id on $I \times h_{i}\left(\left(B_{2}^{n_{i}}-\check{B}^{n_{i}}\right) \times Q\right)$. Extending $g_{1}$ via the identity we get an element $\widetilde{g}_{1} \in$ $C\left(X_{i}\right)$. Note that by choosing $h$ sufficiently close to id we can make $\widetilde{g}_{1}$ as close to id as we please.

By choosing $\widetilde{g}_{1}$ sufficiently close to id and by using the local contractibility of $C\left(X_{i}\right)$, we can find an isotopy $f_{t}: I \times X_{i} \rightarrow I \times X_{i}$ such that $f_{0}=\tilde{g}_{1}, f_{1}=$ id, $f_{t}$ depends continuously on $\widetilde{g}_{1}$, and if $\widetilde{g}_{1}=$ id, then $f_{t}=$ id for each $t$. The restriction $\theta_{t}=f_{t}\left(\mathrm{id} \times h_{i}\right) \mid: I \times \stackrel{\circ}{B}_{2}^{n_{i}} \times Q \rightarrow I \times X_{i}$ gives us an isotopy of open embeddings such that $\left.\theta_{0}=\theta_{1}=\left(\mathrm{id} \times h_{i}\right)\right)$ and $\theta_{t}=\mathrm{id} \times h_{i}$ on $0 \times B_{2}^{n_{i}} \times Q$, for each $t$. Using Theorem 2.1 we can choose an isotopy $\tilde{\theta}_{t}: I \times X_{i} \rightarrow I \times X_{i}$ such that $\widetilde{\theta}_{0}=\widetilde{\theta}_{1}=$ id, $\tilde{\theta}_{t}\left(\right.$ id $\left.\times h_{i}\right)\left|I \times B^{n_{i}} \times Q=\theta_{t}\right| I \times B^{n_{i}} \times Q, \widetilde{\theta}_{t}$ depends continuously on $\theta_{t}$, and $\widetilde{\theta}_{t}$ is supported on $I \times \stackrel{B}{2}_{2}^{n_{i}} \times Q$. Then $\widetilde{\theta}_{t}^{-1} f_{t}: I \times X_{i} \rightarrow I \times X_{i}$ is an isotopy such that $\widetilde{\theta}_{0}^{-1} f_{0}=\widetilde{g}_{1}, \widetilde{\theta}_{1}^{-1} f_{1}=\mathrm{id}$, and $\tilde{\theta}_{t}^{-1} f_{t} \mid I \times h_{i}\left(B^{n i} \times Q\right)=$ id for each $t$. The restriction $\tilde{\theta}_{1}^{-1} f_{t} I \times X_{i-1}: I \times X_{i-1} \rightarrow I \times X_{i-1}$ gives us an isotopy $g_{1} \stackrel{\text { iso }}{\simeq}$ id rel $0 \times X_{i-1}$. The isotopies $h \stackrel{\text { iso }}{\sim} g_{1} \stackrel{\text { iso }}{\simeq}$ id therefore fulfill our requirements.

II. $H(I \times X)$ is locally contractible. Choose any $h \in H(I \times X)$. We need to show that if $h$ is sufficiently close to id, then $h \stackrel{\text { iso }}{\simeq} g$, where $g$ is an element of $C(X)$ such that this isotopy depends continuously on $h$ and if $h=$ id, then each level of this isotopy is the identity. Then using the fact that $C(X)$ is locally contractible this will be enough to imply that $H(I \times X)$ is locally contractible. But the isotopy $h \stackrel{\text { iso }}{\simeq} g \in C(X)$ is just a routine application of Lemmas 3.2 and 3.4 of [3], which follow immediately from [2].

3. Strategy. In this section we will describe how $\$ \S 4$ and 5 are related. The following is the main technical result used in the proof of Theorem 1.

RePRESENTATION THEOREM. Let $M$ be a compact PL manifold, $h$ a $\Delta$ preserving homeomorphism of $\Delta \times I \times M \times Q$ onto itself which is the id on 
$\Delta \times 0 \times M \times Q$, and $g_{0} a \partial \Delta$-preserving homeomorphism of $\partial \Delta \times I \times M$ onto itself such that $h=g_{0} \times$ id on $\partial \Delta \times I \times M \times Q$. Then there exists $a j \geqslant 0$ and a $\Delta$-preserving homeomorphism $\mathrm{g}$ of $\Delta \times I \times M \times B^{j}$ onto itself such that $h \stackrel{\text { iso }}{\cong}(\Delta) g \times$ id $\operatorname{rel}(\Delta \times 0 \times M \times Q) \cup(\partial \Delta \times I \times M \times Q)$.

We remark that for each $j \geqslant 0$ we have a natural splitting $Q=B^{j} \times Q_{j}$, where $Q_{j}=[-1,1]^{\infty}$; thus $g \times \mathrm{id}=g \times \mathrm{id}_{Q_{j}}$. If $d \geqslant 0$ is an integer, we will use $\operatorname{RT}(d)$ to refer to the above statement for all choices of $M, h, g_{0}$ and $\Delta$ such that $\operatorname{dim} \Delta \leqslant d$.

Of crucial importance in the proof of the Representation Theorem is the following result.

Splitting Lemma. Let $M$ be a PL manifold, $\alpha: R^{n} \times B^{k} \rightarrow M$ a PL open embedding, $h: \Delta \times I \times R^{n} \times B^{k} \times Q \rightarrow \Delta \times I \times M \times Q$ a $\Delta$-preserving open embedding such that $h=$ id $\times \alpha \times$ id on $\Delta \times 0 \times R^{n} \times B^{k} \times Q, f_{0}: \partial \Delta \times I \times$ $B_{2}^{n} \times B^{k} \rightarrow \partial \Delta \times I \times M a \partial \Delta$-preserving embedding, and $g_{0}: \partial \Delta \times I \times M \times Q$ $\rightarrow \partial \Delta \times I \times M \times Q a \partial \Delta$-preserving homeomorphism such that $g_{0} h=f_{0} \times$ id on $\partial \Delta \times I \times B_{2}^{n} \times B^{k} \times Q$ and

$$
g_{0}=\mathrm{id} \text { on }(\partial \Delta \times 0 \times M \times Q) \cup\left(\partial \Delta \times I \times M \times Q-h\left(\partial \Delta \times I \times \stackrel{\circ}{B}_{3}^{n} \times B^{k} \times Q\right)\right) \text {. }
$$

Then there exists a $\Delta$-preserving embedding $f: \Delta \times I \times B^{n} \times B^{k} \times B^{j} \rightarrow \cdot \Delta \times I$ $\times M \times B^{j}$ (for some $j \geqslant 0$ ) and a $\Delta$-preserving homeomorphism $g: \Delta \times I \times M \times$ $Q \rightarrow \Delta \times I \times M \times Q$ such that

$$
g h=f \times \text { id on } \Delta \times I \times B^{n} \times B^{k} \times Q, g=g_{0} \text { on } \partial \Delta \times I \times M \times Q,
$$

and

$$
g=\text { id on }(\Delta \times 0 \times M \times Q) \cup\left(\Delta \times I \times M \times Q-h\left(\Delta \times I \times \stackrel{\circ}{B}_{4}^{n} \times B^{k} \times Q\right)\right) \text {. }
$$

If $d \geqslant 0$ is an integer, we will use SL(d) to refer to the above statement for all choices of $M, \alpha, h, f_{0}, g_{0}$, and $\Delta$ such that $\operatorname{dim} \Delta \leqslant d$.

The proof of the Representation Theorem is accomplished by proving $\operatorname{RT}(d)$, for all $d \geqslant 0$, and this is done by inducting on $d$. The case $d=0$ was treated in [7] and the step from $\operatorname{RT}(d-1)$ to $\operatorname{RT}(d)$ is divided into the following two im. plications:

$$
\operatorname{RT}(d-1) \Rightarrow \operatorname{SL}(d) \Rightarrow \operatorname{RT}(d)
$$

The first implication is proved in Theorem 4.1 and the second is proved in Theorem 5.1.

4. Statement and proof of Theorem 4.1. Before proving Theorem 4.1 we will need four lemmas. The first two are established in [7] while the third and 
fourth need some argument. In particular, Lemma 4.1 follows from Lemma 2.3 and Theorem 2 of [7] and Lemma 4.2 follows from Theorem 2 of [7]. Note also that Lemma 4.1 is a strengthened SL(0).

Lemma 4.1. Let $M$ be a PL manifold, $\alpha: R^{n} \times B^{k} \rightarrow M$ be a PL open embedding, and let $h: I \times R^{n} \times B^{k} \times Q \rightarrow I \times M \times Q$ be an open embedding such that $h=\alpha \times$ id on $0 \times R^{n} \times B^{k} \times Q$. Then there exists an embedding $f: I \times B_{2}^{n} \times B^{k} \times B^{j} \rightarrow I \times M \times B^{j}$ (for some $j \geqslant 0$ ) and a homeomorphism $g: I \times M \times Q \rightarrow I \times M \times Q$ such that $g h=f \times$ id on $I \times B_{2}^{n} \times B^{k} \times Q, g=$ id on $(0 \times M \times Q) \cup\left(I \times M \times Q-h\left(I \times B_{3}^{n} \times B^{k} \times Q\right)\right)$, and

$$
\begin{aligned}
h\left(I \times B^{n} \times B^{k} \times Q\right) & \subset f\left(I \times \stackrel{\circ}{B}_{2}^{n} \times B^{k} \times B^{j}\right) \times Q_{j} \\
& \subset f\left(I \times B_{2}^{n} \times B^{k} \times B^{j}\right) \times Q_{j} \subset h\left(I \times \stackrel{\circ}{B}_{3}^{n} \times B^{k} \times Q\right) .
\end{aligned}
$$

LemMA 4.2. Let $M$ be a compact PL manifold, $\alpha: R^{n} \times B^{k} \rightarrow M$ a $P L$ open embedding, $f: I \times R^{n} \times B^{k} \rightarrow I \times M$ a PL open embedding such that $f=\alpha$ on $0 \times R^{n} \times B^{k}$, and $h: I \times M \times Q \rightarrow I \times M \times Q$ a homeomorphism such that $h=$ id on $0 \times M \times Q$ and $h(\mathrm{id} \times \alpha \times$ id $)=f \times$ id on $I \times B_{2}^{n} \times B^{k} \times Q$. Then there exists a PL homeomorphism $g: I \times M \times B^{j} \rightarrow I \times M \times B^{j}$ (for some $j \geqslant 0$ ) such that $g=\mathrm{id}$ on $0 \times M \times B^{j}$ and $g(\mathrm{id} \times \alpha \times \mathrm{id})=f \times$ id on $I \times B^{n} \times$ $B^{k} \times B^{j}$.

In the next two lemmas we will prove some consequences of $\operatorname{RT}(d)$ which will be needed in the proof of Theorem 4.1.

Lemma 4.3. Let $M$ be a compact PL manifold, $K$ a finite simplicial complex such that $\operatorname{dim} K \leqslant d$, and $h: K \times I \times M \times Q \rightarrow K \times I \times M \times Q$ a K-preserving homeomorphism such that $h=\mathrm{id}$ on $K \times 0 \times M \times Q$. Then $\operatorname{RT}(d)$ implies that there exists a K-preserving homeomorphism $g: K \times I \times M \times B^{j} \rightarrow$ $K \times I \times M \times B^{j}($ for some $j \geqslant 0)$ such that $h \stackrel{\text { iso }}{=}(K) g \times$ id rel $K \times 0 \times M \times Q$.

Proof. We induct on $d$. For $d=0$ the result is easy, so passing to the inductive step let $\operatorname{dim} K=d$ and let $K_{d-1}$ be the $(d-1)$-skeleton of $K$. We are going to apply the inductive hypothesis to $K_{d-1}$, but first we have to modify $h$. It is easy to see that $h \stackrel{\text { iso }}{=}(K) h_{1}$ rel $K \times 0 \times M \times Q$, where $h_{1}=h$ on $K_{d-1} \times$ $I \times M \times Q$ and for each $d$-simplex $\sigma$ of $K, h_{1} \mid \sigma \times I \times M \times Q$ is a "product" on a neighborhood of $\partial \sigma \times I \times M \times Q$. By this we mean that there exists a PL open embedding $\theta: \partial \sigma \times[0,1) \rightarrow \sigma$ such that $\theta(x, 0)=x$ and such that

$$
h_{1}(\theta(x, u), t, m, q)=h_{1}(x, t, m, q) \text {, }
$$

for all $x, u, t, m, q$. (We call $\theta$ a PL collaring of $\partial \sigma$.)

Applying the inductive hypothesis and using the above "product" property 
we get $h_{1} \stackrel{\text { iso }}{\sim}(K) h_{2}$ rel $K \times 0 \times M \times Q$, where $h_{2}=g_{0} \times$ id on $K_{d-1} \times I \times M \times Q$ and $g_{0}: K_{d-1} \times I \times M \times B^{j_{1}} \rightarrow K_{d-1} \times I \times M \times B^{j_{1}}$ is a $K_{d-1}$-preserving homeomorphism (for some $j_{1} \geqslant 0$ ). Then in order to finish the inductive step we apply $\operatorname{RT}(d)$ to each $\sigma \times I \times M \times Q$, for $\sigma$ a $d$-simplex of $K$.

LEMMA 4.4. Let $\operatorname{dim} \Delta=d, f: \partial \Delta \times I \times \stackrel{\circ}{B}_{1.5}^{n} \times B^{k} \rightarrow \partial \Delta \times I \times B_{2}^{n} \times$ $B^{k}$ be a $\partial \Delta$-preserving open embedding such that $f=$ id on $\partial \Delta \times 0 \times \stackrel{\circ}{B}_{1.5}^{n} \times B^{k}$, and let $h: \partial \Delta \times I \times B_{2}^{n} \times B^{k} \times Q \rightarrow \partial \Delta \times I \times B_{2}^{n} \times B^{k} \times Q$ be a $\partial \Delta$-preserving homeomorphism such that $h=$ id on $\partial \Delta \times 0 \times B_{2}^{n} \times B^{k} \times Q$ and $h=f \times$ id on $\partial \Delta \times I \times \stackrel{\circ}{B}_{1.5}^{n} \times B^{k} \times Q$. Then $\mathrm{RT}(d-1)$ implies that there exists a $\partial \Delta$-preserving homeomorphism $g$ of $\partial \Delta \times I \times B_{2}^{n} \times B^{k} \times B^{j}$ onto itself (for some $j \geqslant 0$ ) such that

$$
g=\mathrm{id} \text { on } \partial \Delta \times 0 \times B_{2}^{n} \times B^{k} \times B^{j}, g=f \times \operatorname{id} \text { on } \partial \Delta \times I \times B^{n} \times B^{k} \times B^{j},
$$

and

$$
h \stackrel{\text { iso }}{\simeq}(\partial \Delta) g \times \mathrm{id} \operatorname{rel}\left(\partial \Delta \times 0 \times B_{2}^{n} \times B^{k} \times Q\right) \cup\left(\partial \Delta \times I \times B^{n} \times B^{k} \times Q\right) .
$$

Proof. Write $\partial \Delta=\Delta_{1} \cup \Delta_{2}$ where $\Delta_{1}, \Delta_{2}$ are $(d-1)$ simplexes and $\Delta_{1} \cap \Delta_{2}=\partial \Delta_{1}=\partial \Delta_{2}$. Let $\widetilde{\Delta}_{1}$ be a regular neighborhood of $\Delta_{1}$ in $\partial \Delta_{\text {. Then }}$ $\widetilde{\Delta}_{1}$ is a $(d-1)$-simplex containing $\Delta_{1}$ in its interior.

For each $t \in \widetilde{\Delta}_{1}$ we can use [9] to find a neighborhood $U \subset \widetilde{\Delta}_{1}$ of $t$ and a $U$-preserving homeomorphism $\psi_{U}: U \times I \times B_{k}^{n} \times B^{k} \rightarrow U \times I \times B_{.2}^{n} \times B^{k}$ such that $\psi_{U}=$ id on $U \times 0 \times B_{2}^{n} \times B^{k},\left(\psi_{U}\right)_{t}=$ id, and $\left(\psi_{U}\right)_{s} f_{t}=f_{s}$ on $I \times$ $B_{1.4}^{n} \times B^{k}$, for all $s \in U$. Using [12] we can retriangulate $I \times B_{2}^{n} \times B^{k}$ so that $f_{t} \mid I \times \stackrel{\circ}{B}_{1.4}^{n} \times B^{k}$ is a PL open embedding. Then Lemma 4.2 implies that there exists a homeomorphism $\phi$ of $I \times B_{2}^{n} \times B^{k} \times B^{j_{1}}$ onto itself (for some $j_{1} \geqslant 0$ ) such that $\phi=$ id on $0 \times B_{2}^{n} \times B^{k} \times B^{j_{1}}$ and $\phi=f_{t} \times$ id on $I \times B_{1.3}^{n} \times B^{k} \times$ $B^{j_{1}}$. Define $\theta_{U}$ from $U \times I \times B_{2}^{n} \times B^{k} \times B^{j_{1}}$ onto itself by $\left(\theta_{U}\right)_{s}=\left(\left(\psi_{U}\right)_{s} \times \mathrm{id}\right) \phi$, for all $s \in U$. Then $\theta_{U}$ is a $U$-preserving homeomorphism such that $\theta_{U}=$ id on $U \times 0 \times B_{2}^{n} \times B^{k} \times B^{j_{1}}$ and $\left(\theta_{U}\right)_{s}=f_{s}$ on $I \times B_{1.3}^{n} \times B^{k} \times B^{j_{1}}$, for all $s \in U$. Since $\widetilde{\Delta}_{1}$ is contractible we can choose $U=\widetilde{\Delta}_{1}$. To see this one just reduces the problem to that of finding a cross-section for a locally-trivial bundle with $\widetilde{\Delta}_{1}$ as the base space. Put $\theta=\theta_{\widetilde{\Delta}_{1}}$.

Note that $\tilde{h}=(\theta \times \mathrm{id})^{-1} h$ defines a $\widetilde{\Delta}_{1}$-preserving homeomorphism of $\widetilde{\Delta}_{1} \times I \times B_{2}^{n} \times B^{k} \times Q$ onto itself which is the identity on $\left(\widetilde{\Delta}_{1} \times 0 \times B_{2}^{n} \times B^{k}\right.$ $\times Q) \cup\left(\widetilde{\Delta}_{1} \times I \times B_{1.3}^{n} \times B^{k} \times Q\right)$. Applying Lemma 4.3 to $I \times M=\left(I \times B_{2}^{n} \times\right.$ $\left.B^{k}\right)-\left(I \times \stackrel{\circ}{B}_{1.3}^{n} \times B^{k}\right)$, where $0 \times M$ is identified with $\left(0 \times\left(B_{2}^{n}-\stackrel{\circ}{B}_{1.3}^{n}\right) \times B^{k}\right) \cup$ $\left(I \times \partial B_{1.3}^{n} \times B^{k}\right)$, we can find a $\widetilde{\Delta}_{1}$-preserving homeomorphism $\bar{h}$ of $\widetilde{\Delta}_{1} \times I \times$ $B_{2}^{n} \times B^{k} \times Q$ onto itself such that 


$$
\begin{aligned}
& \stackrel{\text { iso }}{\sim}\left(\tilde{\Delta}_{1}\right) \tilde{h} \operatorname{rel}\left(\tilde{\Delta}_{1} \times 0 \times B_{2}^{n} \times B^{k} \times Q\right) \cup\left(\tilde{\Delta}_{1} \times I \times B_{1.3}^{n} \times B^{k} \times Q\right) \\
& U\left(\partial \widetilde{\Delta}_{1} \times I \times B_{2}^{n} \times B^{k} \times Q\right)
\end{aligned}
$$

and $\bar{h}=g_{1} \times$ id on $\Delta_{1} \times I \times B_{2}^{n} \times B^{k} \times Q$, where $g_{1}$ is a $\Delta_{1}$-preserving homeomorphism of $\Delta_{1} \times I \times B_{2}^{n} \times B^{k} \times B^{i 2}$ onto itself (for some $j_{2} \geqslant 0$ ). Note that to get $\bar{h} \stackrel{\text { iso }}{\sim}\left(\widetilde{\Delta}_{1}\right) \widetilde{h}$ rel $\partial \widetilde{\Delta}_{1} \times I \times B_{2}^{n} \times B^{k} \times Q$ we must first modify $\widetilde{h}$ so that it is a "product" on a neighborhood of $\partial \widetilde{\Delta}_{1} \times I \times B_{2}^{n} \times B^{k} \times Q$. (See the construction of $h_{1}$ in the proof of Lemma 4.3.)

Then $(\theta \times$ id $) \bar{h}$ defines a $\widetilde{\Delta}_{1}$-preserving homeomorphism of $\widetilde{\Delta}_{1} \times I \times B_{2}^{n} \times$ $B^{k} \times Q$ onto itself such that

$$
\begin{gathered}
(\theta \times \text { id }) \bar{h}=\text { id on } \tilde{\Delta}_{1} \times 0 \times B_{2}^{n} \times B^{k} \times Q,(\theta \times \text { id }) \bar{h}=h \text { on } \partial \tilde{\Delta}_{1} \times I \times B_{2}^{n} \times B^{k} \times Q, \\
(\theta \times \text { id }) \bar{h}=(\theta \times \text { id })\left(g_{1} \times \text { id }\right) \text { on } \Delta_{1} \times I \times B_{2}^{n} \times B^{k} \times Q, \\
(\theta \times \text { id }) \bar{h}=f \times \text { id on } \widetilde{\Delta}_{1} \times I \times B_{1.3}^{n} \times B^{k} \times Q .
\end{gathered}
$$

Then $(\theta \times$ id $) \bar{h}$ extends by $h$ to a $\partial \Delta$-preserving homeomorphism $h_{1}$ of $\partial \Delta \times I \times$ $B_{2}^{n} \times B^{k} \times Q$ onto itself.

Note that

$$
h_{1} \stackrel{\text { iso }}{\rightleftharpoons}(\partial \Delta) h \operatorname{rel}\left(\partial \Delta \times 0 \times B_{2}^{n} \times B^{k} \times Q\right) \cup\left(\partial \Delta \times I \times B_{1.3}^{n} \times B^{k} \times Q\right)
$$

and if $j_{2}$ is chosen greater than $j_{1}$, then $h_{1}=(\theta \times$ id $) g_{1} \times$ id on $\Delta_{1} \times I \times B_{2}^{n} \times$ $B^{k} \times Q$, where $(\theta \times$ id $) g_{1}$ is a $\Delta_{1}$-preserving homeomorphism of $\Delta_{1} \times I \times B_{2}^{n} \times$ $B^{k} \times B^{j_{2}}$ onto itself. To finish the proof we repeat the above argument for $h_{1} \mid \Delta_{2} \times I \times B_{2}^{n} \times B^{k} \times Q$. This again requires the use of [9], but now instead of using Lemma 4.3 we appeal directly to $\operatorname{RT}(d-1)$.

We are now ready for the proof of Theorem 4.1.

THEOREM 4.1. $\operatorname{RT}(d-1)$ implies SL(d), for any $d \geqslant 1$.

Proof. Working inductively we may assume that $\operatorname{SL}(d-1)$ is true. (Note that Lemma 4.1 implies SL(0).) Consider the data of the statement of SL(d) with $\operatorname{dim} \Delta=d$. We will show how to construct our required $f$ and $g$. Note that the statement of $\operatorname{SL}(d)$ requires that we stabilize by crossing with $B^{j}$, for some appropriately-chosen value of $j \geqslant 0$. In the course of the proof we will have to invoke several results, each of which requires a stabilization. In retrospect no generality is lost if we ignore these stabilizations until the last line of the proof. Adopting this policy for the proof of Theorem 4.1 will simplify the notation and should cause no confusion for the wary reader.

Using Theorem 2.1 we can choose a subdivision of $\Delta$ so fine that if $\sigma$ is a simplex of $\Delta$ and $v$ is a point of $\sigma$, then there exists a $\sigma$-preserving homeomorphism 
$\psi$ of $\sigma \times I \times M \times Q$ onto itself such that

(1) $h_{s}\left(I \times B_{3.5}^{n} \times B^{k} \times Q\right) \subset h_{t}\left(I \times \stackrel{\circ}{3}_{3.6}^{n} \times B^{k} \times Q\right)$, for all $s, t \in \sigma$,

(2) $h_{s}\left(I \times B_{3.8}^{n} \times B^{k} \times Q\right) \subset h_{t}\left(I \times \stackrel{\circ}{B}_{4}^{n} \times B^{k} \times Q\right)$, for all $s, t \in \sigma$,

(3) $\psi_{v}=\mathrm{id}$,

(4) $\psi_{t}=$ id on $(0 \times M \times Q) \cup\left(I \times M \times Q-h_{t}\left(I \times \stackrel{\circ}{B}_{1.5}^{n} \times B^{k} \times Q\right)\right)$, for all $t \in \sigma$,

(5) $\psi_{t} h_{v}=h_{t}$ on $I \times B_{1.4}^{n} \times B^{k} \times Q$, for all $t \in \sigma$.

Let $\bar{\Delta}$ be the $(d-1)$-skeleton of $\Delta$. Using SL $(d-1)$ (which follows from our inductive assumption) we can inductively choose a $\bar{\Delta}$-preserving embedding $\bar{f}$ of $\bar{\Delta} \times I \times B_{1.5}^{n} \times B^{k}$ into $\bar{\Delta} \times I \times M$ and a $\bar{\Delta}$-preserving homeomorphism $\bar{g}$ of $\bar{\Delta} \times I \times M \times Q$ onto itself such that

$$
\bar{g} h=\bar{f} \times \text { id on } \bar{\Delta} \times I \times B_{1.5}^{n} \times B^{k} \times Q, \bar{g}=g_{0} \text { on } \partial \Delta \times I \times M \times Q,
$$

and

$$
\bar{g}=\text { id on }(\bar{\Delta} \times 0 \times M \times Q) \cup\left(\bar{\Delta} \times I \times M \times Q-h\left(\bar{\Delta} \times I \times \stackrel{\circ}{B}_{3.5}^{n} \times B^{k} \times Q\right)\right) \text {. }
$$

Now consider a fixed $d$-simplex $\sigma$ of $\Delta$. We will show how to extend $\bar{f} \mid \partial \sigma \times I \times B^{n} \times B^{k}$ to a $\sigma$-preserving embedding $f: \sigma \times I \times B^{n} \times B^{k} \rightarrow \sigma \times I$ $\times M$ and how to extend $\bar{g} \mid \partial \sigma \times I \times M \times Q$ to a $\sigma$-preserving homeomorphism $g$ : $\sigma \times I \times M \times Q \rightarrow \sigma \times I \times M \times Q$ so that our requirements are satisfied.

Using Lemma 4.1 we can find an open embedding $\theta: I \times R^{n} \times B^{k} \rightarrow I \times M$ such that $\theta=\alpha$ on $0 \times B_{3.7}^{n} \times B^{k}$ and

$$
\begin{aligned}
h_{v}\left(I \times B_{3.6}^{n} \times B^{k} \times Q\right) & \subset \theta\left(I \times \stackrel{\circ}{B}_{3.7}^{n} \times B^{k}\right) \times Q \\
& \subset \theta\left(I \times B_{3.7}^{n} \times B^{k}\right) \times Q \subset h_{v}\left(I \times \stackrel{\circ}{B}_{3.8}^{n} \times B^{k} \times Q\right),
\end{aligned}
$$

(2) $\theta\left(I \times \partial B_{3.7}^{n} \times B^{k}\right) \times Q \hookrightarrow h_{v}\left(\left(I \times B_{3.8}^{n}-B_{3.6}^{n}\right) \times B^{k} \times Q\right)$ is a homotopy equivalence. Let $\tau_{1}$ be the homeomorphism of

$$
\begin{aligned}
A= & \left(0 \times\left(B_{3.7}^{n}-\stackrel{\circ}{B}_{1.4}^{n}\right) \times B^{k} \times Q\right) \cup\left(I \times \partial B_{3.7}^{n} \times B^{k} \times Q\right) \\
& \cup\left(I \times \partial B_{1.4}^{n} \times B^{k} \times Q\right)
\end{aligned}
$$

onto

$$
\begin{aligned}
B= & (\alpha \times \mathrm{id})\left(0 \times\left(B_{3.7}^{n}-B_{1.4}^{n}\right) \times B^{k} \times Q\right) \cup(\theta \times \mathrm{id})\left(I \times \partial B_{3.7}^{n} \times B^{k} \times Q\right) \\
& \cup h_{v}\left(I \times \partial B_{1.4}^{n} \times B^{k} \times Q\right)
\end{aligned}
$$

defined by $\tau_{1}=(\alpha \times$ id $) \cup(\theta \times$ id $) \cup h_{v}$. Clearly there exists a homeomorphism of $A \times[0,1]$ onto $I \times\left(B_{3.7}^{n}-B_{1.4}^{n}\right) \times B^{k} \times Q$ which sends each $(a, 0)$ to a. It is also true that there exists a homeomorphism of $B \times[0,1]$ onto

$$
(\theta \times \mathrm{id})\left(I \times B_{3.7}^{n} \times B^{k} \times Q\right)-h_{v}\left(I \times B_{1.4}^{n} \times B^{k} \times Q\right)
$$


which sends each $(b, 0)$ to $b$. To see this one first uses the fact that the homotopy equivalence

$$
B C(\theta \times \text { id })\left(I \times B_{3.7}^{n} \times B^{k} \times Q\right)-h_{v}\left(I \times B_{1.4}^{n} \times B^{k} \times Q\right)
$$

is homotopic to a homeomorphism (see [6]) to construct a homeomorphism of $B \times[0,1]$ onto (*), and then the homeomorphism extension theorem for $Z$-sets is used to adjust this homeomorphism so that $(b, 0)$ is taken to $b$ (see [8]). Then $\tau_{1}$ extends to a homeomorphism $\tau$ of $I \times B_{3.7}^{n} \times B^{k} \times Q$ onto $\theta\left(I \times B_{3.7}^{n} \times B^{k}\right) \times Q$ such that $\tau=h_{v}$ on $I \times B_{1.4}^{n} \times B^{k} \times Q$.

Note that $\bar{g} \psi($ id $\times \tau)$ gives a $\partial \sigma$-preserving homeomorphism of $\partial \sigma \times I \times B_{3.7}^{n}$ $\times B^{k} \times Q$ onto $\partial \sigma \times \theta\left(I \times B_{3.7}^{n} \times B^{k}\right) \times Q$ which is id $\times \alpha \times$ id on $\partial \sigma \times 0 \times$ $B_{3.7}^{n} \times B^{k} \times Q$, id $\times \theta \times$ id on $\partial \sigma \times I \times \partial B_{3.7}^{n} \times B^{k} \times Q$, and is $\bar{f} \times$ id on $\partial \sigma \times$ $I \times B_{1.4}^{n} \times B^{k} \times Q$. Then (id $\times \theta^{-1} \times$ id) $\bar{g} \psi(\mathrm{id} \times \tau)$ gives a $\partial \sigma$-preserving homeomorphism of $\partial \sigma \times I \times B_{3.7}^{n} \times B^{k} \times Q$ onto itself.

Let $\phi: \partial \sigma \times[0,1) \rightarrow \sigma$ be a PL collaring of $\partial \sigma$ and let $\sigma_{1 / 2}=\sigma-$ $\phi(\partial \sigma \times[0,1 / 2))$. Define $f:\left(\sigma-\dot{\circ}_{1 / 2}\right) \times I \times B^{n} \times B^{k} \rightarrow\left(\sigma-\dot{\circ}_{1 / 2}\right) \times I \times M$ by $f(\phi(x, s), t, y, z)=\bar{f}(x, t, y, z)$. Using Lemma 4.4 we have a $\partial \sigma$-preserving homeomorphism $b$ of $\partial \sigma \times I \times B_{3.7}^{n} \times B^{k}$ onto itself such that

$$
\begin{gathered}
b=\text { id on }\left(\partial \sigma \times 0 \times B_{3.7}^{n} \times B^{k}\right) \cup\left(\partial \sigma \times I \times \partial B_{3.7}^{n} \times B^{k}\right), \\
b \times \text { id }=\left(\mathrm{id} \times \theta^{-1} \times \mathrm{id}\right)(\bar{f} \times \mathrm{id}) \text { on } \partial \sigma \times I \times B^{n} \times B^{k} \times Q,
\end{gathered}
$$

and

(id $\left.\times \theta^{-1} \times \mathrm{id}\right) \bar{g} \psi(\mathrm{id} \times \tau) \stackrel{\text { iso }}{\simeq}(\partial \sigma) b \times \mathrm{id} \operatorname{rel}\left(\partial \sigma \times 0 \times B_{3.7}^{n} \times B^{k} \times Q\right)$

$$
U\left(\partial \sigma \times I \times \partial B_{3.7}^{n} \times B^{k} \times Q\right) \cup\left(\partial \sigma \times I \times B^{n} \times B^{k} \times Q\right) .
$$

Let $b_{s}$ be the above isotopy parametrized so that $0 \leqslant s \leqslant 1 / 2$,

$$
b_{0}=\left(\mathrm{id} \times \theta^{-1} \times \mathrm{id}\right) \bar{g} \psi(\mathrm{id} \times \tau) \text {, and } b_{1 / 2}=b \times \mathrm{id} \text {. }
$$

Define a $\left(\sigma-\stackrel{\circ}{\sigma}_{1 / 2}\right)$-preserving homeomorphism $g$ of $\left(\sigma-{\stackrel{\circ}{\sigma_{1 / 2}}}\right) \times I \times M \times Q$ onto itself by

$$
g_{\phi(x, s)}\left|\theta\left(I \times B_{3.7}^{n} \times B^{k}\right) \times Q=(\theta \times \mathrm{id})\left(b_{s}\right)_{x} \tau^{-1} \psi_{\phi(x, s)}^{-1}\right| \theta\left(I \times B_{3.7}^{n} \times B^{k}\right) \times Q,
$$

and $g_{\phi(x, s)}=$ id elsewhere. Then we have $g=\bar{g}$ on $\partial \sigma \times I \times M \times Q$ and $g h=$ $f \times$ id on $\left(\sigma-\stackrel{\circ}{\sigma}_{1 / 2}\right) \times I \times B^{n} \times B^{k} \times Q$.

Finally we have to show how to define $f \mid \sigma_{1 / 2} \times I \times B^{n} \times B^{k}$ and $g \mid \sigma_{1 / 2} \times$ $I \times M \times Q$. It is easy to extend $b$ to a $\sigma$-preserving homeomorphism $\tilde{b}$ of $\sigma \times I$ $\times B_{3.7}^{n} \times B^{k}$ onto itself such that $\tilde{b}=$ id on $\left(\sigma \times 0 \times B_{3.7}^{n} \times B^{k}\right) \cup(\sigma \times I \times$ $\left.\partial B_{3.7}^{n} \times B^{k}\right)$. We will use $\widetilde{b}$ to get our required extensions of $f$ and $g$.

Let $\beta: \sigma_{1 / 2} \rightarrow \sigma$ be the unique radially-defined homeomorphism which is linear on each ray emanating from the origin. Define $g \mid \sigma_{1 / 2} \times \theta\left(I \times B_{3.7}^{n} \times B^{k}\right) \times$ $Q$ by $g_{x}=(\theta \times$ id $)\left(\widetilde{b}_{\beta(x)} \times\right.$ id $) \tau^{-1} \psi_{x}^{-1}$ and $g_{x}=$ id elsewhere. Note that for $x \in \sigma_{1 / 2}$ we have 


$$
g_{x} h_{x}\left|I \times B^{n} \times B^{k} \times Q=(\theta \times \mathrm{id})\left(\widetilde{b}_{\beta(x)} \times \mathrm{id}\right)\right| I \times B^{n} \times B^{k} \times Q
$$

Then all we have to do is define $f \mid \sigma_{1 / 2} \times I \times B^{n} \times B^{k}$ by $f_{x}=\theta \widetilde{b}_{\beta(x)}$.

5. Statement and proof of Theorem 5.1. The following lemma isolates the key inductive step in the proof of Theorem 5.1.

Lemma 5.1. Let $M$ be a compact PL manifold, $N \subset \partial M$ a compact PL submanifold, $\phi: N \times[0,1) \rightarrow M a$ PL collaring of $N, \alpha: R^{n} \times B^{k} \rightarrow N$ a PL open embedding, $h: \Delta \times M \times Q \rightarrow \Delta \times M \times Q$ a $\Delta$-preserving homeomorphism (where $\operatorname{dim} \Delta \leqslant d), g_{0}: \partial \Delta \times M \rightarrow \partial \Delta \times M a \partial \Delta$-preserving homeomorphism such that $h=g_{0} \times$ id on $\partial \Delta \times M \times Q$, and let $g: \Delta \times M_{1 / 2} \rightarrow \Delta \times M$ be a $\Delta$-preserving embedding (where $M_{t}=M-\phi\left(N \times[0, t)\right.$ ) such that $h=g \times$ id on $\Delta \times M_{1 / 2} \times Q$. Then SL(d) implies that there exists a $\Delta$-preserving homeomorphism $\tilde{h}: \Delta \times M \times$ $Q \rightarrow \Delta \times M \times Q$ and $a \Delta$-preserving embedding

$$
\tilde{g}: \Delta \times\left(M_{2 / 3} \cup \phi\left(\alpha\left(B^{n} \times B^{k}\right) \times[0,2 / 3]\right)\right) \times B^{j} \rightarrow \Delta \times M \times B^{j}
$$

(for some $j \geqslant 0)$ such that $h=\widetilde{g} \times$ id on $\Delta \times\left(M_{2 / 3} \cup \phi\left(\alpha\left(B^{n} \times B^{k}\right) \times[0,2 / 3]\right)\right.$ ) $\times Q$ and $\tilde{h} \stackrel{\text { iso }}{\sim}(\Delta) h \operatorname{rel}\left(\Delta \times M_{2 / 3} \times Q\right) \cup(\partial \Delta \times M \times Q)$.

Proof. Just as in the proof of Lemma 4.4 we can find a $\Delta$-preserving homeomorphism $\theta$ of $\Delta \times M$ onto itself such that $\theta=g$ on $\Delta \times M_{2 / 3}$. Then $(\theta \times \mathrm{id})^{-1} h$ is a $\Delta$-preserving homeomorphism of $\Delta \times M \times Q$ onto itself which is the identity on $\Delta \times M_{2 / 3} \times Q$ and which is $(\theta \times \text { id })^{-1}\left(g_{0} \times\right.$ id) on $\partial \Delta \times M$ $\times Q$. Applying $\operatorname{SL}(d)$ we can get a $\Delta$-preserving embedding

$$
f: \Delta \times \phi\left(\alpha\left(B^{n} \times B^{k}\right) \times[0,2 / 3]\right) \times B^{j} \rightarrow \Delta \times \phi(N \times[0,2 / 3]) \times B^{j}
$$

(for some $j \geqslant 0)$ and a $\Delta$-preserving homeomorphism $\beta$ of $\Delta \times \phi(N \times[0,2 / 3]) \times$ $Q$ onto itself such that

$$
\beta(\theta \times \text { id })^{-1} h=f \times \text { id on } \Delta \times \phi\left(\alpha\left(B^{n} \times B^{k}\right) \times[0,2 / 3]\right) \times Q
$$

and

$$
\begin{aligned}
\beta= & \text { id on }(\partial \Delta \times \phi(N \times[0,2 / 3]) \times Q) \cup(\Delta \times \phi(N \times 2 / 3) \times Q) \\
& \cup\left(\Delta \times \phi(N \times[0,2 / 3]) \times Q-(\theta \times \mathrm{id})^{-1} h\left(\Delta \times \phi\left(\alpha\left(\stackrel{\circ}{2}_{2}^{n} \times B^{k}\right) \times[0,2 / 3]\right) \times Q\right)\right)
\end{aligned}
$$

Our required $\Delta$-preserving homeomorphism $\tilde{h}$ is defined by $\tilde{h}=h$ on $\Delta \times$ $M_{2 / 3} \times Q$ and $\tilde{h}=(\theta \times \mathrm{id}) \beta(\theta \times \mathrm{id})^{-1} h$ on $\Delta \times \phi(N \times[0,2 / 3]) \times Q$. It follows from the contractibility of $H(I \times Q, 0 \times Q)$, the group of all homeomorphisms of $I \times Q$ which are fixed on $0 \times Q$, that $\widetilde{h} \stackrel{\text { iso }}{=}(\Delta) h \operatorname{rel}\left(\Delta \times M_{2 / 3} \times Q\right) \cup$ $(\partial \Delta \times M \times Q)$. Our required $\Delta$-preserving embedding $\widetilde{g}$ is defined by $\widetilde{g}=g \times$ id on $\Delta \times M_{2 / 3} \times B^{j}$ and $\tilde{g}=(\theta \times$ id $) f$ on $\Delta \times \phi\left(\alpha\left(B^{n} \times B^{k}\right) \times[0,2 / 3]\right) \times B^{j}$. 
THEOREM 5.1. SL(d) implies $\mathrm{RT}(d)$, for any $d \geqslant 1$.

Proof. Just as in the proof of Theorem 4.1 we will ignore the stabilizations necessary to carry out the constructions in this proof. Choose a filtration $M_{0} \subset M_{1} \subset \cdots \subset M_{p}=M$ of $M$, where $M_{0}$ is a regular neighborhood of $\partial M$ and each $M_{i}$ is obtained from $M_{i-1}$ by attaching a PL handle. For each $i$ let $\widetilde{M}_{i}=([0,1 / 2] \times M) \cup\left([1 / 2,1] \times M_{i}\right)$ thus giving a filtration $\tilde{M}_{0} \subset \tilde{M}_{1} \subset \cdots \subset$ $\widetilde{M}_{p}=I \times M$. Observe that for each $i$ there exists a compact PL submanifold $N_{i}$ of $\partial(I \times M)$, a PL collaring $\phi_{i}: N_{i} \times[0,1) \rightarrow I \times M$, and a PL open embedding $\alpha_{i}: R^{n_{i}} \times B^{k_{i}} \rightarrow N_{i}$ such that $\widetilde{M}_{i}=I \times M-\phi_{i}\left(N_{i} \times[0,2 / 3)\right)$ and $\tilde{M}_{i+1}=\tilde{M}_{i}$ $\cup \phi_{i}\left(\alpha_{i}\left(B^{n_{i}} \times B^{k}\right) \times[0,2 / 3]\right)$. We also note that there exists a PL homeomorphism of $I \times M$ onto itself taking $\widetilde{M}_{0}$ onto $[0,1 / 3] \times M$. Thus we may assume that $\widetilde{M}_{0}=[0,1 / 3] \times M$.

We will inductively carry out the following construction: For each $i$ there exists a regular neighborhood $\bar{M}_{i}$ of $\widetilde{M}_{i}$, a $\Delta$-preserving embedding $\bar{g}_{i}: \Delta \times \bar{M}_{i} \rightarrow$ $\Delta \times I \times M$ and a $\Delta$-preserving homeomorphism $h_{i}$ of $\Delta \times I \times M \times Q$ onto itself such that $h_{i}=\bar{g}_{i} \times$ id on $\Delta \times \bar{M}_{i} \times Q, h_{i}=g_{0} \times$ id on $\partial \Delta \times I \times M \times Q$, and $h_{i} \stackrel{\text { iso }}{\simeq}(\Delta) h \mathrm{rel}(\Delta \times 0 \times M \times Q) \cup(\partial \Delta \times I \times M \times Q)$. It is clear that this will be sufficient to prove Theorem 5.1.

We first consider the case $i=0$. Let us regard $\Delta$ as $B_{1}^{d}$, where $\Delta_{t}=B_{t}^{d}$. Note that $h \stackrel{\text { iso }}{=}(\Delta) h^{\prime} \operatorname{rel}(\Delta \times 0 \times M \times Q) \cup(\partial \Delta \times I \times M \times Q)$, where for each $x \in \Delta-\AA_{1 / 2}$ and radial projection $x^{\prime}$ of $x$ onto $\partial \Delta$ we have $h_{x}^{\prime}=h_{x^{\prime}}$. Let $\theta$ : $\Delta \rightarrow[0,1 / 2]$ be defined by $\theta(x)=1 / 2$, for $x \in \Delta_{1 / 2}$, and $\theta(x)=1-t$, for $x \in \partial \Delta_{t}$ and $1 / 2 \leqslant t \leqslant 1$. Then there exists a $\Delta$-preserving homeomorphism $h_{0}$ of $\Delta \times I \times$ $M \times Q$ onto itself defined by $\left(h_{0}\right)_{x}=$ id on $[0, \theta(x)] \times M \times Q$ and on $[\theta(x), 1]$ $\times M \times Q$ we just let $\left(h_{0}\right)_{x}$ be $h_{x}^{\prime}$ linearly scaled-down from $[0,1] \times M \times Q$ to $[\theta(x), 1] \times M \times Q$. Clearly $h_{0} \stackrel{\text { iso }}{\approx}(\Delta) h \operatorname{rel}(\Delta \times 0 \times M \times Q) \cup(\partial \Delta \times I \times M \times$ Q). To get our desired $\bar{g}_{0}: \Delta \times \bar{M}_{0} \rightarrow \Delta \times I \times M$ (where $\left.\bar{M}_{0}=[0,1 / 2] \times M\right)$ we define $\left(\bar{g}_{0}\right)_{x}=$ id on $[0, \theta(x)] \times M$, for all $x \in \Delta$, and to get $\left(g_{0}\right)_{x} \mid[\theta(x), 1 / 2] \times \cdot M$, for $x \in \Delta-\AA_{1 / 2}$, we first linearly scale-down $\left(g_{0}\right)_{x}$ from $[0,1] \times M$ to $[\theta(x), 1]$ $\times M$ and then suitably restrict it. Then $h_{0}=\bar{g}_{0} \times$ id on $\Delta \times \bar{M}_{0} \times Q$.

Passing to the inductive step let us assume that $\bar{M}_{i}, \bar{g}_{i}$, and $h_{i}$ have been constructed. Without loss of generality we may assume that $\bar{M}_{i} \supset I \times M-$ $\phi_{i}\left(N_{i} \times[0,1 / 2)\right) . \quad$ Put

$$
\bar{M}_{i+1}=\left[I \times M-\phi_{i}\left(N_{i} \times[0,7 / 12)\right)\right] \cup \phi_{i+1}\left(\alpha_{i+1}\left(B_{2}^{n_{i+1}} \times B^{k_{i+1}}\right) \times[0,7 / 12]\right)
$$

and use Lemma 5.1 to get a $\Delta$-preserving embedding $\bar{g}_{i+1}$ of $\Delta \times \bar{M}_{i+1}$ into $\Delta \times$ $I \times M$, and a $\Delta$-preserving homeomorphism $h_{i+1}$ of $\Delta \times I \times M \times Q$ onto itself such that $h_{i+1}=g_{0} \times$ if on $\partial \Delta \times I \times M \times Q$ and $h_{i+1} \simeq(\Delta) h_{i} \operatorname{rel}(\Delta \times 0 \times M \times Q)$ $U(\partial \Delta \times I \times M \times Q)$. 
6. Proof of Theorem 1. Assuming the data of Theorem 1 we will first need to know that $\pi_{n} C(M \times Q)$ is isomorphic to the direct limit of the system (*) $\pi_{n} \mathrm{C}(M) \stackrel{(\times \mathrm{id})_{*}}{\longrightarrow} \pi_{n} \mathrm{C}(M \times[-1,1]) \stackrel{(\times \mathrm{id})_{*}}{\longrightarrow} \pi_{n} \mathrm{C}\left(M \times[-1,1]^{2}\right) \rightarrow \cdots$. Call this direct limit $\pi_{n}^{\infty} \mathrm{C}(M)$ and note that there is a natural homomorphism $\theta$ : $\pi_{n}^{\infty} C(M) \rightarrow \pi_{n} C(M \times Q)$ which is induced by the commutativity of

$$
\pi_{n} C\left(M \times[-1,1]^{i}\right) \stackrel{(\times \mathrm{id})_{*}}{\longrightarrow} \pi_{n} C\left(M \times[-1,1]^{i+1}\right)
$$

for each $i \geqslant 0$.

It follows from the Representation Theorem that $\theta$ is $1-1$ and it follows from Lemma 4.3 that $\theta$ is onto. Thus we have $\pi_{n}^{\infty} C(M) \approx \pi_{n} C(M \times Q)$. It is worth remarking that this result is independent of the results of [10]. On the other hand if we use the results of [10] we see that the system (*) stabilizes, i.e. for some $i \geqslant 0$ (dependent on $n$ ) we have

$$
\pi_{n} C\left(M \times[-1,1]^{i}\right) \stackrel{(\times \mathrm{id})}{\approx} \pi_{n} \mathrm{C}\left(M \times[-1,1]^{i+1}\right) \approx \cdots .
$$

This implies that $\pi_{n} C\left(M \times[-1,1]^{\dagger}\right) \approx \pi_{n}^{\infty} C(M)$.

7. Proof of Theorem 4. In [7] the following exact sequence was lished for $X$ any compact $Q$-manifold.

$$
\cdots \rightarrow \pi_{n} C(X) \rightarrow \pi_{n} H(X) \stackrel{\theta}{\rightarrow} \pi_{n} E(X) \rightarrow \cdots \rightarrow \pi_{0} E(X) .
$$

The map $\theta$ is induced by the inclusion $f i(X) \hookrightarrow E(X)$. The proof of (*) given in [7] uses [3], but Theorem 2.2 of the present paper removes that dependence on [3]. It is clear that Theorem 4 follows from (*) and the Corollary of Theorem 3.

\section{REFERENCES}

1. D. Burghelea and D. W. Henderson, Smoothings and homeomorphisms for Hilbert manifolds, Bull. Amer. Math. Soc. 76 (1970), 1261-1265. MR 44 \#1057.

2. T. A. Chapman, Canonical extensions of homeomorphisms, General Topology and Appl. 2 (1972), 227-247. MR 46 \#9924. (1973), 227-239.

3., Homeomorphisms of Hilbert cube manifolds, Trans. Amer. Math. Soc. 182

4. - Homotopic homeomorphisms of infinite-dimensional manifolds, Compositio Math. 27 (1973), 135-140. MR 49 \#4014,

5. - Compact Hubert cube manifolds and the invariance of Whitehead torsion, Bull. Amer. Math. Soc. 79 (1973), 52-56. $448-497$.

6. - Topological invariance of Whitehead torsion, Amer. J. Math. 96 (1974), 
7. T. A. Chapman, Homotopic homeomorphisms of Hilbert cube manifolds, Lecture Notes in Math., vol. 438, ,Springer-Verlag, Berlin and New York, 1975, pp. 122-136.

8. Notes on Q-manifolds, University of Kentucky, 1973 (preprint).

9. R. D. Edwards and R. C. Kirby, Deformations of spaces of imbeddings, Ann. of Math. (2) 93 (1971), 63-88. MR 44 \#1032.

10. A. E. Hatcher, Higher simple homotopy theory. I, II (preprint).

11. A. E. Hatcher and J. Wagoner, Pseudo-isotopies of compact manifolds, Asterisque 6 (1973).

12. R. C. Kirby, Lectures on triangulation of manifolds, UCLA Lecture notes, 1969. 13. C. P. Rourke, Embedded handle theory, concondance and isotopy, Topology of Manifolds (Proc. Inst. Univ. of Georgia, Athens, Ga., 1969), Markham, Chicago, Ill., 1970, pp. 431-438. MR 43 \#5537.

DEPARTMENT OF MATHEMATICS, UNIVERSITY OF KENTUCKY, LEXINGTON, KENTUCKY 40503 